\section{Commentary: Radiographic stroke: The silent killer?}

\author{
Bradley G. Leshnower, MD
}

Since Griepp and colleagues' initial report ${ }^{1}$ of arch replacement using deep hypothermic circulatory arrest, cerebralprotection strategies have rapidly evolved. In the last decade, there has been an increase in use of moderate hypothermic circulatory arrest (MHCA) with antegrade cerebral perfusion (ACP) via the right axillary (RA) or innominate artery (IA). Despite multiple retrospective reports on the safety of IA cannulation for ACP, the site of arterial cannulation for ACP has never been rigorously investigated. This month's report on the Aortic Surgery Cerebral Protection Evaluation (ACE) trial answers this question. ${ }^{2}$

This Canadian multicenter trial randomized 111 patients undergoing proximal aortic surgery with hemiarch replacement to either RA or IA cannulation for the delivery of ACP during the period of MHCA (nasopharyngeal temperature $25.7^{\circ} \mathrm{C}$ ). Patients received pre- and postoperative diffusion-weighted magnetic resonance imaging (DWMRI) of the brain as well as postoperative neurocognitive and serum biomarker assessments. The overall results were excellent, with a $<4 \%$ mortality rate and a reported incidence of stroke/transient ischemic attack of 5.4\%, although neurologic examinations were performed by the surgeon alone. It has been demonstrated that postcardiac surgery adverse neurologic events are underreported when adjudicated by the surgeon as opposed to a neurologist. ${ }^{3}$ There were no significant differences in operative time, mortality, stroke/transient ischemic attack, radiographic or serum markers of neurologic injury, or neurocognitive outcomes between the 2 different methods of cannulation for ACP. Based on these results, the authors concluded that

From the Division of Cardiothoracic Surgery, Emory University School of Medicine, Atlanta, Ga.

Disclosures: The author reported no conflicts of interest.

The Journal policy requires editors and reviewers to disclose conflicts of interest and to decline handling or reviewing manuscripts for which they may have a conflict of interest. The editors and reviewers of this article have no conflicts of interest.

Received for publication Nov 8, 2020; revisions received Nov 8, 2020; accepted for publication Nov 11, 2020; available ahead of print Nov 21, 2020.

Address for reprints: Bradley G. Leshnower, MD, Division of Cardiothoracic Surgery, Department of Surgery, Emory University School of Medicine, 1365 Clifton Rd NE, Suite A 2257, Atlanta, GA 30322 (E-mail: bleshno@emory.edu).

J Thorac Cardiovasc Surg 2022;164:1442-3

$0022-5223 / \$ 36.00$

Copyright (c) 2020 by The American Association for Thoracic Surgery

https://doi.org/10.1016/j.jtcvs.2020.11.032

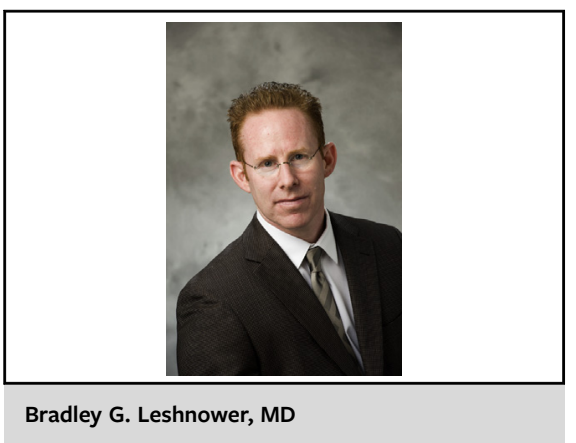

CENTRAL MESSAGE

The incidence of MRI-

adjudicated neurologic injury is

high following hemiarch

replacement. The clinical signifi-

cance of this injury remains

poorly understood.

ACP with IA cannulation is safe and affords equivalent neuroprotection as ACP with RA cannulation.

The primary finding of the ACE trial is the confirmation of safety and efficacy of IA cannulation for ACP. However, an equally important product of this trial is the information gleaned from the DW-MRI data. Despite a low incidence of clinically evident adverse neurologic events, $69 \%$ of patients with IA and $59 \%$ of patients with RA had new postoperative ischemic strokes by DW-MRI. Furthermore, by the authors' definitions, $39 \%$ of patients with RA and $34 \%$ of patients with IA had new severe ischemic brain lesions postoperatively. The clinical significance of these silent infarcts is poorly understood; however, total infarct volume by MRI has been correlated with cognitive decline in patients following surgical aortic valve replacement. ${ }^{4}$

A high incidence of postoperative clinically silent MRIadjudicated brain injury following hemiarch replacement was also recently reported in a pilot study that randomized patients to either MHCA + ACP or deep hypothermic circulatory arrest and retrograde cerebral perfusion. ${ }^{5}$ The wide discrepancy observed between clinical and radiographic stroke from both of these trials supports the notion that clinical stroke alone is an inadequate metric to evaluate the efficacy of cerebral protection. Instead, a combination of clinical, radiographic, and neurocognitive data is necessary to comprehensively assess neurologic injury following circulatory arrest operations. The optimal cerebral 
protection for hemiarch replacement is widely debated and has yet to be proven. The path forward is to conduct prospective randomized trials of cerebral-protection strategies that incorporate rigorous, comprehensive neurologic investigations such as the current study.

\section{References}

1. Griepp RB, Stinson EB, Hollingsworth JF, Buehler D. Prosthetic replacement of the aortic arch. J Thorac Cardiovasc Surg. 1975;70:1051-63.
2. Peterson MD, Garg V, Mazer CD, Chu MWA, Bozinovski J, Dagenais F, et al. A randomized trial comparing axillary versus innominate artery cannulation for aortic arch surgery. J Thorac Cardiovasc Surg. 2022;164:1426-38.e2.

3. Messé SR, Acker MA, Kasner SE, Fanning M, Giovannetti T, Ratcliffe SJ, et al. Stroke after aortic valve surgery: results from a prospective cohort. Circulation 2014;129:2253-61.

4. Giovannetti T, Price CC, Fanning M, Messé S, Ratcliffe SJ, Lyon A, et al. Cognition and cerebral infarction in older adults after surgical aortic valve replacement. Ann Thorac Surg. 2019;107:787-94.

5. Leshnower BG, Rangaranju S, Allen JW, Stringer AY, Gleason TG, Chen EP. Deep hypothermia with retrograde cerebral perfusion versus moderate hypothermia with antegrade cerebral perfusion for arch surgery. Ann Thorac Surg. 2019;107:1104-10. 